\title{
The proximity condition
}

\author{
Conal Duddy • Ashley Piggins
}

Received: 20 November 2011 / Accepted: 23 November 2011 / Published online: 14 December 2011 (C) Springer-Verlag 2011

\begin{abstract}
We investigate the social choice implications of what we call "the proximity condition". Loosely speaking, this condition says that whenever a profile moves "closer" to some individual's point of view, then the social choice cannot move "further away" from this individual's point of view. We apply this idea in two settings: merging functions and preference aggregation. The precise formulation of the proximity condition depends on the setting. First, restricting attention to merging functions that are interval scale invariant, we prove that the only functions that satisfy proximity are dictatorships. Second, we prove that the only social welfare functions that satisfy proximity and a version of the Pareto criterion are dictatorships. We conclude that either proximity is not an attractive normative requirement after all, or we must give up some other social choice condition. Another possibility is that our normative intuition about proximity needs to be codified using different axioms.
\end{abstract}

\section{Introduction}

In this paper, we investigate the social choice implications of what we call "the proximity condition". To the best of our knowledge, the condition originates in a paper by MacIntyre (1998) on topological social choice. MacIntyre calls it "monotonicity" but we prefer to call it "proximity". Loosely speaking, this condition says that whenever a profile moves closer to some individual's point of view, then the social choice cannot

\section{Duddy}

Department of Philosophy, Logic and Scientific Method, London School of Economics, London, UK e-mail: conal.duddy@gmail.com

\section{A. Piggins ( $\varangle) \cdot$ C. Duddy}

J.E. Cairnes School of Business and Economics, National University of Ireland Galway, University Road, Galway, Ireland e-mail: ashley.piggins@nuigalway.ie 
move further away from this individual's point of view. We apply this idea in two settings: merging functions and preference aggregation. The precise formulation of the proximity condition depends on the setting. ${ }^{1}$

First, restricting attention to merging functions that are interval scale invariant, we prove that the only functions that satisfy proximity are dictatorships. Second, we prove that the only social welfare functions that satisfy proximity and a version of the Pareto criterion are also dictatorships. We conclude that either proximity is not an attractive normative requirement after all, or we must give up some other social choice condition. Another possibility is that our normative intuition about proximity needs to be codified using different axioms.

Consider first merging. Suppose that we wish to combine a set of numbers into a single number. This single number is intended to "aggregate" or "synthesise" the original set of numbers. Aczél and Roberts (1989) call these procedures "merging functions". They are also known as "aggregation functions". ${ }^{2}$ Examples of merging functions are those generating the arithmetic mean, the geometric mean etc.

Merging functions are germane to social choice theory. An obvious example is public good provision. Members of a group may have conflicting views as to how much of a public good ought to be produced (each individual's view is a number). These numbers need to be combined into a single number, so that a group choice can be made. An application of merging functions to social choice problems can be found in Quesada (2007). Unlike Quesada, we do not restrict attention to a discrete set of numbers. In our model, any real number is admissible. However, like him, we derive an impossibility result: the only merging functions satisfying our axioms are dictatorships.

It is possible to imagine various requirements of merging. The unit of measurement and the zero point, or origin, of the measurement should not matter, for instance. For example, say that a group of economists seek to collectively predict what the level of national debt will be in 12 months' time. They each submit an individual prediction $x_{i}$ and the value taken by the merging function is $x$. So $x$ is the collective prediction. Now suppose that the current level of debt is $d$ and that, instead of submitting $x_{i}$, each economist submits the number $x_{i}-d$, the amount by which he or she believes that the level of debt will change. Then the value taken by the merging function should be $x-d$. Similarly, if they each submit the proportion $\frac{1}{d} x_{i}$ then the outcome should be $\frac{1}{d} x$.

In this paper, we assume that merging satisfies this requirement. We call it "interval scale invariance", following Grabisch et al. (2009). ${ }^{3}$

We add the following proximity axiom. Suppose that everyone moves strictly closer to some individual's view (their number). We can interpret this, in the language of social choice theory, as a change in the "profile" of views. How should this change in the profile influence the group view? Notice that one individual has not changed his opinion at the new profile, but everyone else is now strictly closer to it (where "closer" is measured in terms of absolute distance). A natural interpretation of this

\footnotetext{
1 Baigent (1987) considers the social choice implications of a condition he calls "proximity preservation". This is a continuity-like condition.

2 An extensive treatment is given by Grabisch et al. (2009).

3 Aczél et al.(1994) study functional equations of this kind.
} 
profile change is that everyone else in the group has become more sympathetic to this individual's point of view. It seems reasonable to expect then, as a consequence of the profile change, that the group number (the group view) should either stay where it is or, similarly, move closer to this individual's number. Surely the group choice should not penalise this person now that everyone else has become more sympathetic to their opinion?

Our theorem demonstrates that, for merging functions that are interval scale invariant, this normative requirement is no different from requiring dictatorship (in the standard social choice sense). Given that dictatorship is antithetical to any notion of "merging", we must reject this proximity axiom for merging functions of this type.

The second setting we consider is preference aggregation. For simplicity, we consider a model in which there are three individuals and three alternatives. An obvious problem we encounter when we move to a model of preference aggregation is how to account for "closening" in a profile. We need this in order to reflect our intuition about proximity. Largely due to its familiarity, we adopt Kemeny's well-known measure of distance between preferences. ${ }^{4}$ Individuals 2 and 3 move closer to individual 1 if the Kemeny distance between 2 and 1 and 3 and 1 both fall.

In the model each individual has (exact) strict preferences over the three alternatives and a social planner aggregates these into a single preference. This is keeping in the tradition of classical social choice theory. However, we allow this single preference to be "fuzzy". This seems particularly appropriate for a special issue in honour of Maurice Salles; fuzzy social choice is an area in which Maurice has made many distinguished contributions. ${ }^{5}$ At the technical level, the assumption that the co-domain of a social welfare function is fuzzy might lead one to believe that it is possible to smooth the aggregation of preferences, thus avoiding the impossibility theorems of Arrow (1951) and others. The results of this research project are mixed. ${ }^{6}$ In fact, in this paper we will derive an impossibility result that makes use of the central theorem in Barrett et al. (1986).

Formally, a fuzzy strict preference is a function $h$ from $A^{2}$ to $[0,1]$ where $A$ is the set of alternatives. Different interpretations can be given to $h(a, b)$. We follow Barrett, Pattanaik and Salles in this respect and interpret $h(a, b)$ as a degree of confidence. For us (like them), $h(a, b)$ is the degree to which the social planner is confident that $a$ is better than $b$.

Given this, the interpretation we give to the model is as follows. Imagine that a social planner wishes to discover the "objectively correct" ranking of three social states. In other words, the planner wishes to discover which alternatives are objectively better than which. Suppose that the only information available to the planner is each individual's (exact) strict preferences. The planner uses a social welfare function to aggregate this information. The inputs of the function are (exact) individual strict pref-

\footnotetext{
4 Kemeny and Snell (1962, chapter 2) is a detailed explanation of Kemeny's metric. They provide an axiomatisation of the metric. Duddy and Piggins (2011) also discuss Kemeny's metric.

5 The central paper in the field is Barrett et al. (1986) which we shall draw on in this paper. A recent survey is Barrett and Salles (2011).

6 See Barrett and Salles (2011) for a survey. One issue that arises is how to model the transitivity of a fuzzy binary relation. On this see Salles (1998) and Duddy et al. (2011).
} 
erences, and the output is a fuzzy strict preference. These individual preferences can be interpreted as each individual's view as to which alternatives are objectively better than which. Social preference, however, is fuzzy in order to represent how confident the social planner is that one alternative is better than another given these individual evaluations. $^{7}$

With this interpretation in mind, it is natural to impose two axioms. The first is the Pareto criterion. This says that if everyone believes that $a$ is better than $b$, then the social planner should be completely confident that $a$ is better than $b$. The second axiom is our counterpart of the proximity condition, which we call Proximity*. This condition says the following. Take, for example, two profiles $P, P^{\prime}$ in which individual $i$ 's preference ranking does not change, and in which $i$ prefers $a$ to $b .{ }^{8}$ Furthermore, assume that no individual moves away from $i$ in the move from $P$ to $P^{\prime}$ (in terms of Kemeny distance), and that those preferring $a$ to $b$ at $P$ still do so at $P^{\prime}$. Then, according to Proximity*, the degree to which the social planner is confident that $a$ is preferred to $b$ cannot fall in the move from $P$ to $P^{\prime}$, nor can the degree of confidence that $b$ is preferred to $a$ rise.

One way of justifying Proximity* is as follows. The social planner might feel that the likelihood of individual $i$ holding the objectively correct $(a, b)$ ranking depends on two things: (i) how many people agree with $i$ on this pair, and (ii) how close the other individuals are to $i$ "overall" (Kemeny-wise). If both of these things (weakly) increase, then $i$ is at least as likely as before to be correct about $(a, b)$. Therefore, the social planner will not lower $h(a, b)$ nor will the planner raise $h(b, a)$. This is what Proximity* accomplishes.

We prove that the only social welfare functions that satisfy the Pareto criterion and Proximity* are dictatorships. This means that there is a single individual such that, at every profile and for every pair of alternatives $a$ and $b$, if this individual prefers $a$ to $b$, then the social planner must have some confidence (not zero) that $a$ is better than $b$ and must have zero confidence that $b$ is better than $a$.

As mentioned above, our proof of this theorem depends critically on the central theorem of Barrett et al. (1986).

One conclusion of our model of preference aggregation is that there is a trade-off between different axioms. Either we must give up the Pareto criterion or the Proximity* condition. Another possibility is that our normative intuition about proximity needs to be codified using a different axiom than Proximity*. Such an axiom might, in fact, be compatible with the Pareto condition.

The paper is organised as follows. In Sect. 2 we present a model of merging. Sect. 3 contains our theorem about merging functions. In Sect. 4 we present a model of preference aggregation. Sect. 5 contains our theorem on social welfare functions. Sect. 6 concludes.

\footnotetext{
${ }^{7}$ Barrett et al. (1986, p. 2) note that "even when individual preferences are exact, one may still like to permit non-exact social preferences so as to have a more flexible framework for aggregating individual preferences".

${ }^{8}$ Saying that individual $i$ prefers $a$ to $b$ is equivalent to saying that individual $i$ believes that $a$ is objectively better than $b$.
} 


\section{Merging}

Let $N$ be a finite set $\{1,2, \ldots, n\}$ of individuals with $n \geq 3$. A merging function (or aggregation function) is a function $f: \mathbb{R}^{n} \rightarrow \mathbb{R}$ where $\mathbb{R}$ denotes the real line. Given any $\mathbf{x}, \mathbf{y} \in \mathbb{R}^{n}$, let $\left(x_{1}, \ldots, x_{n}\right)=\mathbf{x},\left(y_{1}, \ldots, y_{n}\right)=\mathbf{y}$ and so on. And let $x$ denote $f(\mathbf{x}), y$ denote $f(\mathbf{y})$ and so on. We write $\mathbf{1}$ for $(1,1, \ldots, 1)$, the vector of $n 1$ 's.

The following are conditions that merging functions may satisfy.

Interval scale invariance. For all $\alpha>0$, all $\beta \in \mathbb{R}$ and all $\mathbf{x} \in \mathbb{R}^{n}$, if $\mathbf{y}=\alpha \mathbf{x}+\beta \mathbf{1}$ then $y=\alpha x+\beta$.

Proximity. For all $\mathbf{x}, \mathbf{y} \in \mathbb{R}^{n}$ and all $i \in N$, if $x_{i}=y_{i}$ and $\left|x_{i}-y_{j}\right|<$ $\left|x_{i}-x_{j}\right|$ for all $j \in N-\{i\}$ then $\left|x_{i}-y\right| \leq\left|x_{i}-x\right|$.

Dictatorship. There exists $i \in N$ such that for all $\mathbf{x} \in \mathbb{R}^{n}, x=x_{i}$.

\section{A theorem about merging}

Lemma 1 says the following. If we can find a profile where everyone has a different opinion and the group choice corresponds to one of these opinions, then at every profile the group choice will always be identical to this individual's opinion (their number).

Lemma 1 Let $f$ be a merging function that is interval scale invariant and satisfies the proximity condition. If there exists $\mathbf{x} \in \mathbb{R}^{n}$ such that $\#\left\{x_{1}, \ldots, x_{n}\right\}=n$ and $x \in\left\{x_{1}, \ldots, x_{n}\right\}$ then $f$ is dictatorial.

Proof Assume that there exists $\mathbf{x} \in \mathbb{R}^{n}$ and $i \in N$ such that $\#\left\{x_{1}, \ldots, x_{n}\right\}=n$ and $x=x_{i}$. Take any $\mathbf{y} \in \mathbb{R}^{n}$.

There must exist $\alpha \in \mathbb{R}$ with $\alpha>0$ such that for all $j \in N-\{i\},\left|x_{i}-x_{j}\right|>$ $\left|\alpha y_{i}-\alpha y_{j}\right|$.

There must also exist $\beta \in \mathbb{R}$ such that $\alpha y_{i}+\beta=x_{i}$. Let $\mathbf{z}=\alpha \mathbf{y}+\beta \mathbf{1}$.

We know by construction that $x_{i}=z_{i}$ and also that $\left|x_{i}-x_{j}\right|>\left|z_{i}-z_{j}\right|$ for all $j \in N-\{i\}$. This inequality holds because the right-hand side (RHS) is equal to $\left|\alpha y_{i}-\alpha y_{j}\right|$.

Proximity implies that $\left|x_{i}-x\right| \geq\left|z_{i}-z\right|$. Given that $x=x_{i}$ and $z_{i}=x_{i}$, we have then $z=x_{i}$.

Given that $\mathbf{z}=\alpha \mathbf{y}+\beta \mathbf{1}$, we have $z=\alpha y+\beta$ since $f$ is interval scale invariant. Since $z=x_{i}$ we have $x_{i}=\alpha y+\beta$. Therefore $y=\left(x_{i}-\beta\right) / \alpha$. We know that $\alpha y_{i}+\beta=x_{i}$ and so we have $y=y_{i}$.

The proximity condition says that if every individual moves closer to individual $k$ 's opinion then the group choice cannot move away from individual $k$. Lemma 2 says the following. Suppose that there is an individual $k$ whose opinion is distinct from the opinions of all of the other individuals. Imagine that individual $j$ unilaterally moves closer to this individual's opinion. Lemma 2 says that the group choice cannot move away from $k$ 's opinion.

Lemma 2 Let $f$ be a merging function that is interval scale invariant and satisfies the proximity condition. For all $j, k \in N$ and all $\mathbf{x}, \mathbf{y} \in \mathbb{R}^{n}$ such that $\mathbf{x}$ and $\mathbf{y}$ differ 
only at the jth component and $x_{k} \neq x_{i}$ for all $i \in N-\{k\}$, if $\left|y_{j}-y_{k}\right|<\left|x_{j}-x_{k}\right|$ then $\left|y-y_{k}\right| \leq\left|x-x_{k}\right|$.

Proof Take any $j, k \in N$ and two $n$-tuples $\mathbf{x}, \mathbf{y} \in \mathbb{R}^{n}$ that differ only at the $j$ th component with $x_{k} \neq x_{i}$ for all $i \in N-\{k\}$.

Assume, by way of contradiction, that $\left|x_{j}-x_{k}\right|>\left|y_{j}-y_{k}\right|$ and yet $\left|x-x_{k}\right|<$ $\left|y-y_{k}\right|$.

Let $\alpha$ be any positive number that is strictly less than 1 but that is sufficiently close to 1 such that $\left|x-x_{k}\right|<\left|\alpha\left(y-y_{k}\right)\right|$.

Consider the profile $\mathbf{z}=\alpha \mathbf{y}+(1-\alpha) y_{k} \mathbf{1}$. Interval scale invariance implies that $z=\alpha y+(1-\alpha) y_{k}$.

Comparing $\mathbf{z}$ with $\mathbf{y}$, note that $z_{k}=y_{k}$ and that $\left|y_{i}-y_{k}\right|>\left|z_{i}-z_{k}\right|$ for all $i \in$ $N-\{j, k\}$. This inequality holds because the RHS is equal to $\left|\left(\alpha y_{i}+(1-\alpha) y_{k}\right)-y_{k}\right|$ which equals $\left|\alpha\left(y_{i}-y_{k}\right)\right|$. Since $0<\alpha<1$ and $y_{i} \neq y_{k}$ for all $i \in N-\{j, k\}$, the RHS must be less than $\left|y_{i}-y_{k}\right|$. It must also be the case that $\left|y_{j}-y_{k}\right| \geq\left|z_{j}-z_{k}\right|$.

Recall that the $\mathbf{x}$ and $\mathbf{y}$ profiles are identical except for the $j$ th component. We have seen that $\left|y_{i}-y_{k}\right|>\left|z_{i}-z_{k}\right|$ for all $i \in N-\{j, k\}$. So then $\left|x_{i}-x_{k}\right|>\left|z_{i}-z_{k}\right|$ for all $i \in N-\{j, k\}$. Recall also that, by assumption, $\left|x_{j}-x_{k}\right|>\left|y_{j}-y_{k}\right|$. We derived above that $\left|y_{j}-y_{k}\right| \geq\left|z_{j}-z_{k}\right|$. It follows from this and our assumption that $\left|x_{j}-x_{k}\right|>\left|y_{j}-y_{k}\right|$ that $\left|x_{j}-x_{k}\right|>\left|z_{j}-z_{k}\right|$. Hence $\left|x_{i}-x_{k}\right|>\left|z_{i}-z_{k}\right|$ for all $i \in N-\{k\}$. Therefore, since $z_{k}=x_{k}$, the proximity condition implies that $\left|x-x_{k}\right| \geq\left|z-z_{k}\right|$.

However, $z-z_{k}$ is equal to $\alpha y+(1-\alpha) y_{k}-z_{k}$. Or, since $z_{k}=y_{k}$, we can write $z-z_{k}=\alpha y+(1-\alpha) y_{k}-y_{k}$. That is, $z-z_{k}=\alpha\left(y-y_{k}\right)$. Therefore, $\left|x-x_{k}\right| \geq\left|z-z_{k}\right|$ implies $\left|x-x_{k}\right| \geq\left|\alpha\left(y-y_{k}\right)\right|$. But we chose $\alpha$ such that $\left|x-x_{k}\right|<\left|\alpha\left(y-y_{k}\right)\right|$ and therefore we have a contradiction.

We are now in a position to state our theorem.

Theorem 3 A merging function that is interval scale invariant satisfies the proximity condition if and only if it is a dictatorship.

Proof Let $f$ be a merging function. The $(\Leftarrow)$ direction is trivial, so we only prove $(\Rightarrow)$.

Take an $\mathbf{x} \in \mathbb{R}^{n}$ with $x_{1}<x_{2}<\ldots<x_{n}$ and $x_{m}=\frac{1}{2}\left(x_{1}+x_{n}\right)$ for some $m \in N$. We consider three cases.

Case 1. $x \in\left\{x_{1}, \ldots, x_{n}\right\}$. Lemma 1 implies that $f$ is dictatorial.

Case 2. $x \notin\left\{x_{1}, \ldots, x_{n}\right\}$ and $\left(x_{1}<x<x_{m}\right.$ or $\left.x_{m}<x<x_{n}\right)$. Assume that $x_{1}<x<x_{m}$ (a similar argument applies when $x_{m}<x<x_{n}$ ). Consider a $\mathbf{y} \in \mathbb{R}^{n}$ that is identical to $\mathbf{x}$ except that $y_{n}=x$. In other words, the $n$th individual's opinion has moved closer to both $x_{1}$ and $x_{m}$.

Lemma 2 implies then that $\left|x-x_{1}\right| \geq\left|y-x_{1}\right|$ and $\left|x-x_{m}\right| \geq\left|y-x_{m}\right|$ which can only be true if $y=x$. Therefore $y_{n}=y$ and Lemma 1 implies that $f$ is dictatorial. This contradicts $x \notin\left\{x_{1}, \ldots, x_{n}\right\}$.

Case 3. $x \notin\left\{x_{1}, \ldots, x_{n}\right\}$ and $\left(x>x_{n}\right.$ or $\left.x<x_{1}\right)$. Assume that $x>x_{n}$ (a similar argument applies when $x<x_{1}$ ). 
Take $x_{1}$, the unique, smallest number in $\left\{x_{1}, \ldots, x_{n}\right\}$. Increase this number in the following way. Make it equal to $2 x_{n}-x_{2}$, leaving all other numbers unchanged. This yields a new profile $\mathbf{x}^{*} \in \mathbb{R}^{n}$.

This increase in the value of $x_{1}$ means that $x_{n}=\frac{1}{2}\left(x_{2}+x_{1}^{*}\right)$.

By construction, the individual whose number has changed has moved closer to $x_{n}$. To see this, note that the previous distance was $\left|x_{n}-x_{1}\right|$ and now it is $\left|x_{1}^{*}-x_{n}\right|=$ $\left|2 x_{n}-x_{2}-x_{n}\right|=\left|x_{n}-x_{2}\right|$. Given that $x_{1}<x_{2}<x_{n}$ the required conclusion follows.

Lemma 2 implies then that $\left|x^{*}-x_{n}\right| \leq\left|x-x_{n}\right|$.

Also, by construction, the individual whose number has changed has moved further away from $x_{2}$. To see this, note that the original distance was $\left|x_{1}-x_{2}\right|$ and now it is $\left|2 x_{n}-x_{2}-x_{2}\right|=\left|2\left(x_{n}-x_{2}\right)\right|$. Since $x_{2} \leq x_{m}$ the required conclusion follows.

Again, Lemma 2 implies that $\left|x-x_{2}\right| \leq\left|x^{*}-x_{2}\right|$.

These two inequalities, and the fact that $x_{2}<x_{n}<x$, means that $x^{*}=x$.

Given that $x_{1}$ has increased, and that the group choice is unchanged, there are two possibilities. Either (i) $x^{*}>x_{1}^{*}$ or (ii) $x_{n}<x^{*} \leq x_{1}^{*}$. If (ii) then Case 1 and Case 2 above imply that $f$ is dictatorial. This contradicts the assumption that $x \notin\left\{x_{1}, \ldots, x_{n}\right\}$. If (i) then we can repeatedly apply the procedure described above (increasing the smallest number in the profile). As we have seen, at each stage, the group choice cannot change. However, eventually we must reach (ii) and so $f$ is dictatorial, contradicting the assumption that $x \notin\left\{x_{1}, \ldots, x_{n}\right\}$.

\section{Preference aggregation}

Let $A$ be a set of three alternatives. The numbers 1, 2 and 3 represent the individuals. The assumption of three alternatives and three individuals is made for simplicity. Let $N=\{1,2,3\}$.

Let $E$ be the set of all (exact) linear orders over $A .{ }^{9}$ A profile is an element of $E^{3}$. We denote profiles by $P=\left(P_{1}, P_{2}, P_{3}\right), P^{\prime}=\left(P_{1}^{\prime}, P_{2}^{\prime}, P_{3}^{\prime}\right)$ and so on.

A fuzzy binary relation (FBR) is a function $h$ from $A^{2}$ to $[0,1]$ such that, for all $a, b, c \in A$, (i) $h(a, a)=0$, (ii) if $h(a, b)=1$ then $h(b, a)=0$, (iii) $h(a, b)>0$ or $h(b, a)>0$, and (iv) if $h(a, b)>0$ and $h(b, c)>0$ then $h(a, c)>0$. Let $H$ be the set of all fuzzy binary relations.

A social welfare function (SWF) is a function from $E^{3}$ to $H$. When discussing a social welfare function, we write $h$ for the value it takes at profile $P$, and $h^{\prime}$ for the value it takes at profile $P^{\prime}$, and so on.

The Kemeny distance between two orderings $P_{i}$ and $P_{j}$ is equal to $\left|P_{i}-P_{j}\right|{ }^{10}$ Given two profiles $P$ and $P^{\prime}$ and two individuals $i, j \in N$, if $\left|P_{i}-P_{j}\right| \geq\left|P_{i}^{\prime}-P_{j}^{\prime}\right|$ we say that in the move from $P$ to $P^{\prime}$ individual $i$ has not moved away from individual $j$. The following is our counterpart of the original proximity condition, this time expressed in the framework of social welfare functions.

\footnotetext{
9 A linear order is an asymmetric, transitive and complete binary relation.

10 Here we write $|W|$ to denote the cardinality of the set $W$.
} 
Proximity*. For all profiles $P$ and $P^{\prime}$, all $i \in N$ and all $a, b \in A$ such that $a P_{i} b$ and $P_{i}=P_{i}^{\prime}$, if (i) in the move from $P$ to $P^{\prime}$ no individual has moved away from individual $i$ and (ii) $a P_{j} b$ implies $a P_{j}^{\prime} b$ for all $j \in N$, then $h(a, b) \leq h^{\prime}(a, b)$ and $h(b, a) \geq h^{\prime}(b, a)$.

This condition says the following. Take, for example, two profiles $P, P^{\prime}$ in which individual $i$ 's preference ranking does not change, and in which $i$ prefers $a$ to $b$. Furthermore, assume that no individual moves away from $i$ in the move from $P$ to $P^{\prime}$ (in terms of Kemeny distance), and that those preferring $a$ to $b$ at $P$ still do so at $P^{\prime}$. Then, according to Proximity*, the degree to which the social planner is confident that $a$ is preferred to $b$ cannot fall in the move from $P$ to $P^{\prime}$, nor can the degree of confidence that $b$ is preferred to $a$ rise.

The following conditions are more familiar. They are special cases of the Barrett et al. (1986) conditions applied to a domain of (exact) linear orders.

Fuzzy Independence (FI). For all profiles $P$ and $P^{\prime}$ and all $a, b \in A$, if $a P_{i} b \leftrightarrow a P_{i}^{\prime} b$ for all $i \in N$ then $h(a, b)=h^{\prime}(a, b)$.

Pareto criterion (PC). For all $a, b \in A$ and all profiles $P$, if $a P_{i} b$ for all $i \in N$ then $h(a, b)=1$.

Dictatorship*. There exists $i \in N$ such that, for all profiles $P$ and all $a, b \in A, h(a, b)>0$ if and only if $a P_{i} b$.

\section{A theorem about preference aggregation}

It is clear that PC and FI are logically independent. We can confirm that Proximity* implies neither PC nor FI by considering the very simple social welfare function defined as follows.

For all profiles $P$, if $P_{1}=P_{2}=P_{3}$ then, for all $a, b \in A, h(a, b)=1$ if $a P_{1} b$, and $h(a, b)=0$ otherwise. If it is not the case that $P_{1}=P_{2}=P_{3}$ then, for all $a, b \in A$, $h(a, b)=0.5$.

This simple function violates both PC and FI. However, it satisfies Proximity*. To see this, suppose that, in the move from one profile to another, individuals 1 and 2 have not moved away from individual 3. Then there are only two possibilities: either the social FBR is unchanged or else it is entirely determined by individual 3.

Proximity*, FI and PC can all be satisfied by a simple pairwise majority rule, although this requires the co-domain of the SWF to be extended. Let $T$ be the set of all functions $t$ from $A^{2}$ to $[0,1]$ such that, for all $a, b \in A$, (i) $t(a, a)=0$, (ii) if $t(a, b)=1$ then $t(b, a)=0$ and (iii) $t(a, b)>0$ or $t(b, a)>0$. Define a function from $E^{3}$ to $T$ as follows. For all profiles $P$ and all $a, b \in A, t(a, b)$ equals one if at least two individuals prefer $a$ to $b$, and it equals zero otherwise. This rule satisfies the three conditions Proximity*, FI and PC and is clearly not dictatorial. However, some or all of the functions in $T-H$ may be difficult to interpret as representations of a social planner's degree of confidence. Henceforth we restrict our attention to SWFs that map into $H$.

We have seen that Proximity* does not imply FI or PC. However, the conjunction of Proximity* and PC does imply FI. 
Lemma 4 If a social welfare function satisfies Proximity* and PC then it satisfies FI.

Proof Consider any social welfare function $h$ that satisfies Proximity* and PC, and take any $a, b \in A$. We will restrict our attention to profiles $P, P^{\prime}$ at which $a P_{i} b \leftrightarrow a P_{i}^{\prime} b$ for all $i \in N$. First, consider those profiles in which all three individuals prefer $a$ to $b$. It follows immediately from PC that $h(a, b)=1$ at all such profiles. Conversely, when all three individuals prefer $b$ to $a$ then $h(a, b)=0$.

We have to prove that FI holds in the absence of unanimity over $a$ and $b$. Without loss of generality, let us assume that $P, P^{\prime}$ are such that individuals 1 and 2 prefer $a$ to $b$ while individual 3 prefers $b$ to $a$.

We employ Saari's geometry of voting in our proof. ${ }^{11}$

In an equilateral triangle identify each vertex with an alternative and define a binary relationship of a point in terms of its proximity to a vertex. Thus, point $p$ corresponds to the ranking $a \succ b$ if and only if $p$ is closer to vertex $a$ than to vertex $b .^{12}$

This relationship subdivides the equilateral triangle where the open regions (the smallest triangles) correspond to strict rankings without indifference among the alternatives, while the line segments and the barycentric point correspond to rankings with indifference. Given that we only consider strict preferences, we can ignore all of these latter points.

Of course, points in each open region correspond to the same preference ranking. Points in region i correspond to the ranking $c \succ a \succ b$. Points in region ii correspond to the ranking $a \succ c \succ b$, and so on. To illustrate which regions correspond to which rankings, the numbering in Fig. 1 comes from the Table 1.

In our model there are just three individuals labelled 1,2, and 3. Therefore, to represent a profile, we put the individuals in the corresponding region of the representation triangle. To illustrate, consider the following figure.

In the profile represented by Fig. 2, individual 1 has the preference ranking $c \succ$ $b \succ a$, individual 2 has the preference ranking $b \succ a \succ c$, and individual 3 has the preference ranking $a \succ c \succ b$.

Fig. 1 The representation triangle

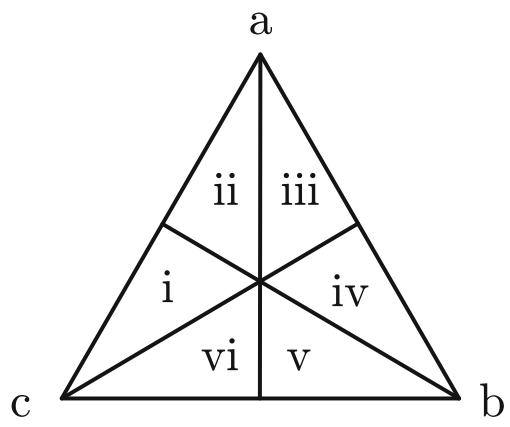

\footnotetext{
11 For an introduction to Saari's geometry, we recommend Saari (1995, 2001). Perote-Peña and Piggins (2002) give a simple proof of Arrow's impossibility theorem using this geometry.

$12 a \succ b$ denotes that $a$ is preferred to $b$.
} 
Table 1 Numbers assigned to preference rankings

\begin{tabular}{ll}
\hline Number & Ranking \\
\hline i & $c \succ a \succ b$ \\
ii & $a \succ c \succ b$ \\
iii & $a \succ b \succ c$ \\
iv & $b \succ a \succ c$ \\
v & $b \succ c>a$ \\
vi & $c \succ b \succ a$ \\
\hline
\end{tabular}

Fig. 2 A profile

C

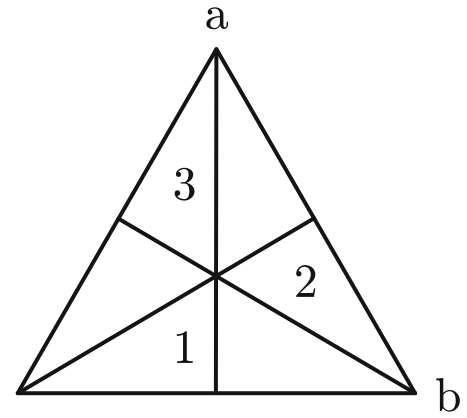

For the purposes of our proof, we will use, in addition to Saari's standard representation triangle, truncated versions of the triangle. An example of a truncated triangle is given in Fig. 3.

This triangle is truncated because the $\{a, c\}$ line segment has been omitted.

A truncated representation triangle represents a truncated profile; that is, individual 1 has the preference ranking $a \succ b \succ c$, but all we know about individual 3 is that she ranks $b \succ c$ and $b \succ a$, but her $\{a, c\}$ ranking is unspecified. Similarly, individual 2 ranks $a \succ b$ and $c \succ b$, but her $\{a, c\}$ ranking is also unspecified.

Recall that we assume that $P, P^{\prime}$ are such that individuals 1 and 2 prefer $a$ to $b$ while individual 3 prefers $b$ to $a$.

This means that there are 27 possible profiles consistent with these $\{a, b\}$ rankings. Four of these profiles are compatible with Fig. 3.

Fig. 3 A truncated representation triangle

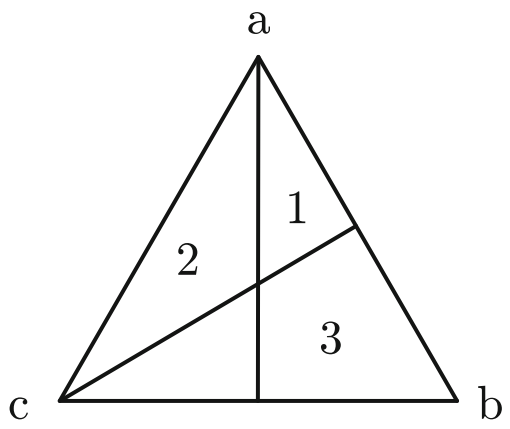


Each of the 27 possible profiles is compatible with one of the triangles in Fig. 4.

Note that there is only one non-truncated profile in this figure. This is represented by the northernmost of the two triangles in the centre of the figure. At this profile, individuals 1 and 2 have the $a \succ b \succ c$ ranking, and individual 3 ranks $c \succ b \succ a$. We will call this the "central profile".

Next, we introduce the concept of a "completion". A completion takes the profile space in Fig. 4 and completes it in an arbitrary way. By this we mean that each truncated triangle is turned into a standard representation triangle that is compatible with it. Moreover, a completion must be "consistent" in a sense that we describe below.

In order to explain this concept, take an arbitrary truncated triangle.

For simplicity, we will choose the triangle in the north east corner of Fig. 4. This represents the following truncated profile. Individual 1 ranks $c \succ b$ and $a \succ b$ but her $\{a, c\}$ ranking is unspecified. Individual 2 has the ranking $a \succ b \succ c$. Individual 3 ranks $b \succ c$ and $b \succ a$ but her $\{c, a\}$ ranking is unspecified.

In a completion, each of these unspecified pairwise rankings is made specific. For example, in one possible completion, individual 1 ranks $a \succ c$ (and so holds a $a \succ c \succ b$ ranking) and individual 3 ranks $c \succ a$ (and so holds a $b \succ c \succ a$ ranking).

Crucially, the way the rankings are "completed" must be consistent across triangles. So, given that we have imposed an $a \succ c$ ranking on individual 1 , in all truncated triangles in which individual 1's $\{a, c\}$ ranking is unspecified, we must impose $a \succ c$

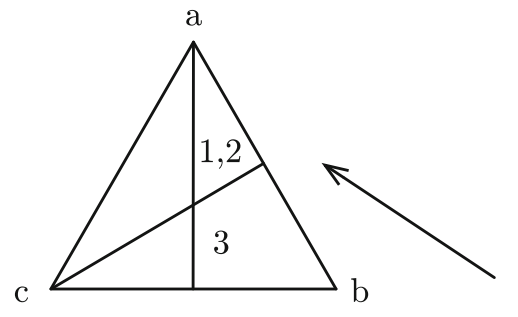

c

c
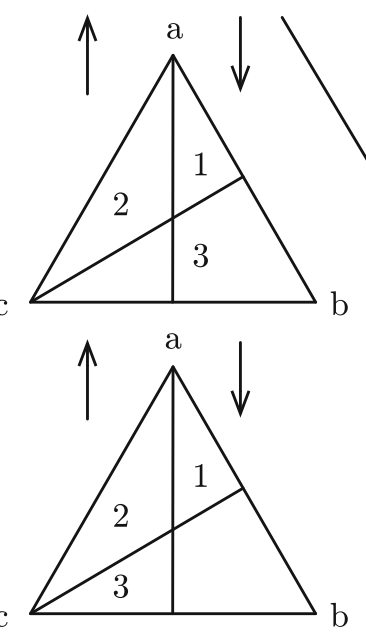

C c
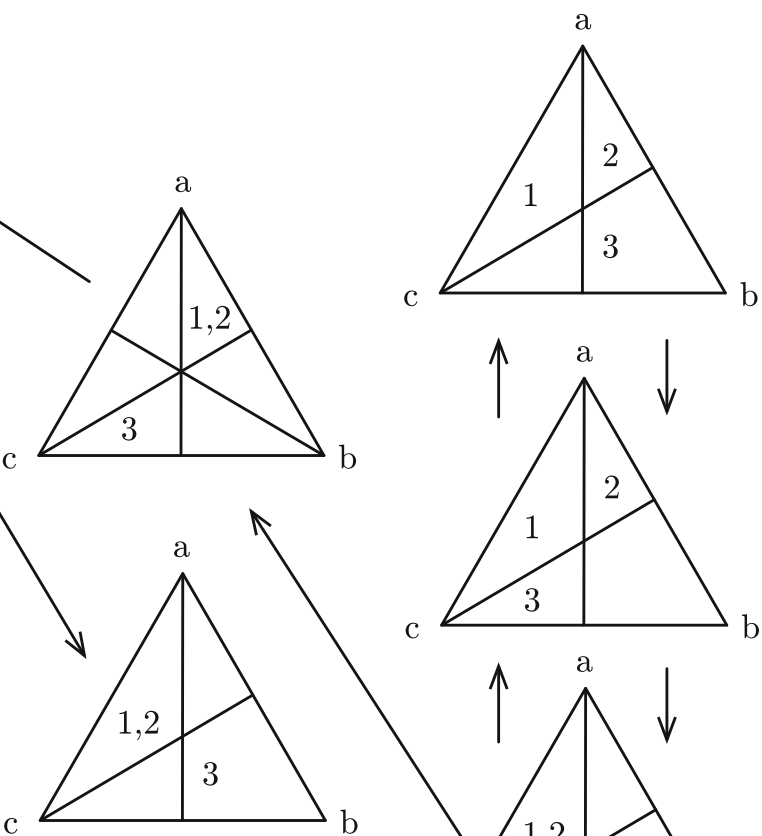

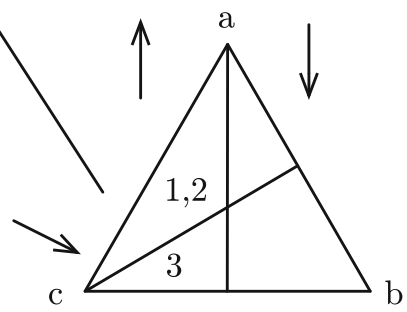

Fig. 4 Profile space 
too. Similarly, given that we have imposed a $c \succ a$ ranking on individual 3, in all truncated triangles in which individual 3's $\{c, a\}$ ranking is unspecified, we must impose $c \succ a$ too.

This process not only turns the truncated triangle in the north east corner of Fig. 4 into a standard representation triangle, but it also "completes" other truncated triangles. For example, the triangle directly beneath this one is completed too. As we can see, imposing a $a \succ c$ ranking on individual 1 turns that triangle into a standard representation triangle (note that individual 2 and 3's preferences are already fully specified in that triangle).

It is important to note that some triangles remain truncated despite the imposition of these $\{a, c\}$ rankings on individuals 1 and 3 at triangles where these $\{a, c\}$ rankings are unspecified for these individuals. The reason for this is that in our original truncated triangle (chosen arbitrarily), individual 2's preferences were completely specified as $a \succ b \succ c$.

As we can see, in other truncated triangles, individual 2's $\{a, c\}$ ranking is unspecified. Therefore, to finish our "completion", we need to impose an $\{a, c\}$ ranking on individual 2 at those triangles in Fig. 4 in which individual 2's $\{a, c\}$ ranking is unspecified. Imagine that we impose the $a \succ c$ ranking on individual 2.

This process yields one logically possible completion.

Figure 5 represents this completion (of course, there are others).

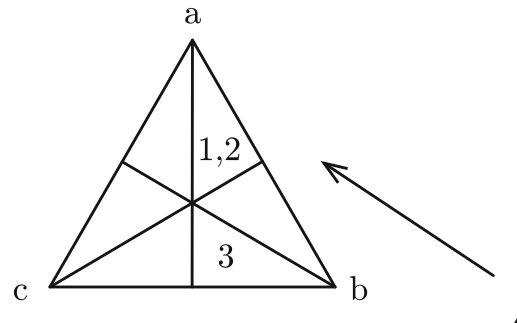

c

c
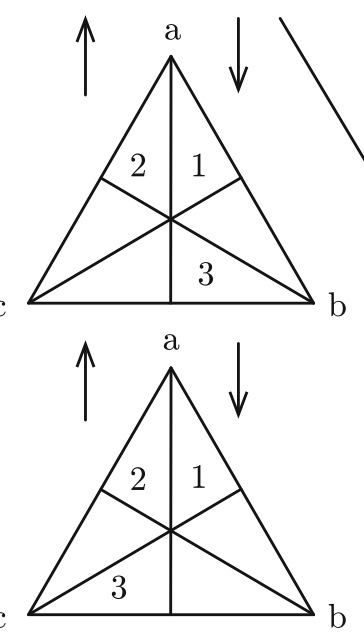

c

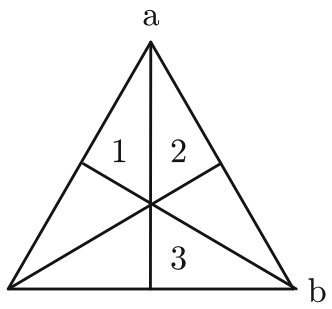

c
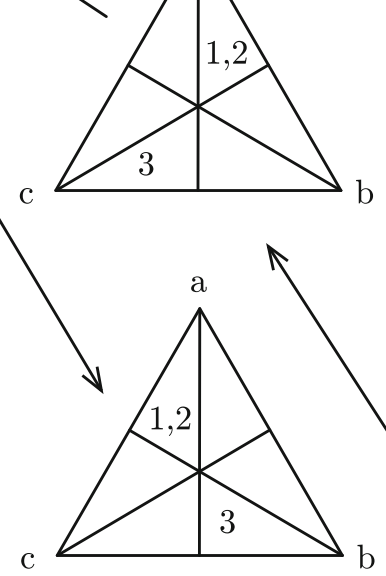
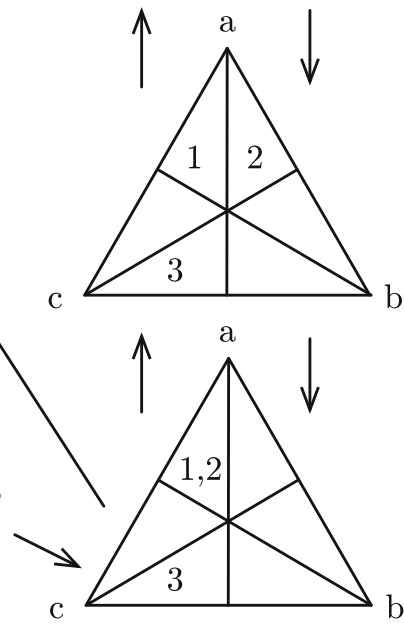

Fig. 5 One possible completion 
Fig. 6 Far left column

C
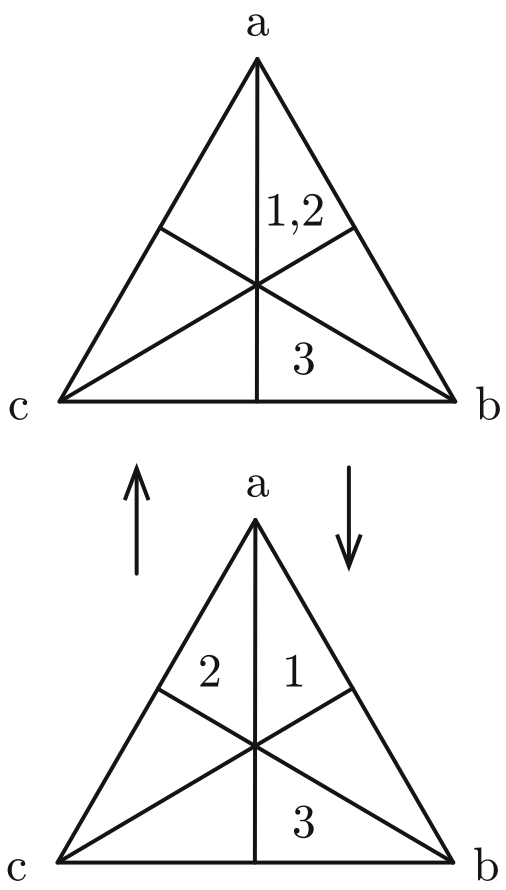

C

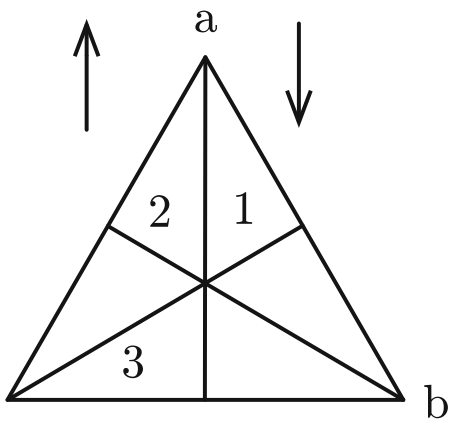

We have drawn arrows that originate from a representation triangle and point towards another. In order to explain these arrows, take the column of three triangles at the far left of Fig. 5. For simplicity, we isolate this column of triangles in the figure below.

Take the middle triangle in Fig. 6. In the move from this profile to the profile directly above it (represented by the top triangle), individual 2 has moved closer to individual 1 (in terms of Kemeny distance). Moreover, individual 3 has not moved away from individual 1 (again, in terms of Kemeny distance). Note too that everyone who ranked $a \succ b$ at the middle profile still does so at the top profile. Applying the Proximity* condition, the value $h$ assigns to $(a, b)$ at the top profile must be at least as great as the value it assigns to $(a, b)$ at the middle profile. 
This is what the arrow represents. We draw an arrow pointing to the top profile from the middle profile to denote that $h(a, b)$ is at least as large at the top profile as it is at the middle profile. Let $P$ denote the profile represented by the middle triangle and $P^{\prime}$ denotes the profile represented by the top triangle. The argument above shows that $h^{\prime}(a, b) \geq h(a, b)$.

Notice, however, that we have also drawn an arrow from the top profile to the middle profile. The reason for this is that in the move from the middle profile to the top profile, individual 2 has also moved closer to individual 3. Moreover, individual 1 has not moved away from individual 3. Note too that everyone who ranked $b \succ a$ at the middle profile still does so at the top profile (this, in fact, is just individual 3 herself). Applying the Proximity* condition, the value $h$ assigns to $(b, a)$ at the top profile must be at least as great as the value it assigns to $(b, a)$ at the middle profile.

However, Proximity* also implies that the value $h$ assigns to $(a, b)$ at the top profile cannot exceed the value it assigns to $(a, b)$ at the middle profile. This is why we have drawn an arrow from the top profile to the middle profile. So $h^{\prime}(a, b) \leq h(a, b)$. Therefore, it must be the case that $h(a, b)=h^{\prime}(a, b)$.

Now let us consider the middle profile and the bottom profile. The latter we denote by $P^{\prime \prime}$. In the move from $P$ to $P^{\prime \prime}$ individual 3 has moved closer to individual 2 , and individual 1 is no further away from individual 2. Proximity* implies that $h^{\prime \prime}(a, b) \geq$ $h(a, b)$. However, in the move from $P^{\prime \prime}$ to $P$ individual 3 moves closer to individual 1 , and individual 2 is no further away from individual 1. Proximity* implies that $h(a, b) \geq h^{\prime \prime}(a, b)$. So we know that $h(a, b)=h^{\prime \prime}(a, b)$.

This proves that the value $h$ assigns to $(a, b)$ must be the same at all three profiles in Fig. 6.

The same analysis applies to the three profiles in the column at the far right of Fig. 5.

The analysis of the profiles in Fig. 7 simply mirrors our treatment of the profiles in Fig. 6. So we can conclude that the value $h$ assigns to $(a, b)$ must be the same at all three profiles in Fig. 7.

The link between these two sets of profiles is provided by what we call the "middle segment". This is the set of profiles in Fig. 8.

For simplicity, we have labelled these profiles; profile 1, 2, 3 and the central profile. The central profile will turn out to be critical for the proof of the lemma (it is the only non-truncated profile in Fig. 4).

Profiles 1 and 3 have already featured in our earlier analysis. Consider the move from profile 2 to 1 . In this move, both individuals 1 and 2 move closer to individual 3. Proximity* implies then that $h^{2}(b, a) \leq h^{1}(b, a)$ and $h^{1}(a, b) \leq h^{2}(a, b)$.

Consider the move from profile 2 to profile 3 . In this move, individual 3 is closer to both individuals 1 and 2. Proximity* implies that $h^{3}(a, b) \geq h^{2}(a, b)$.

Consider the move from the central profile to profile 3 . In this move, both individuals 1 and 2 move closer to individual 3. Proximity* implies that $h^{3}(b, a) \geq h^{\text {central }}(b, a)$ and $h^{\text {central }}(a, b) \geq h^{3}(a, b)$.

Finally, consider the move from the central profile to profile 1 . In this move, individual 3 is closer to both individuals 1 and 2 . Proximity* implies that $h^{1}(a, b) \geq$ $h^{\text {central }}(a, b)$. 
Fig. 7 Far right column
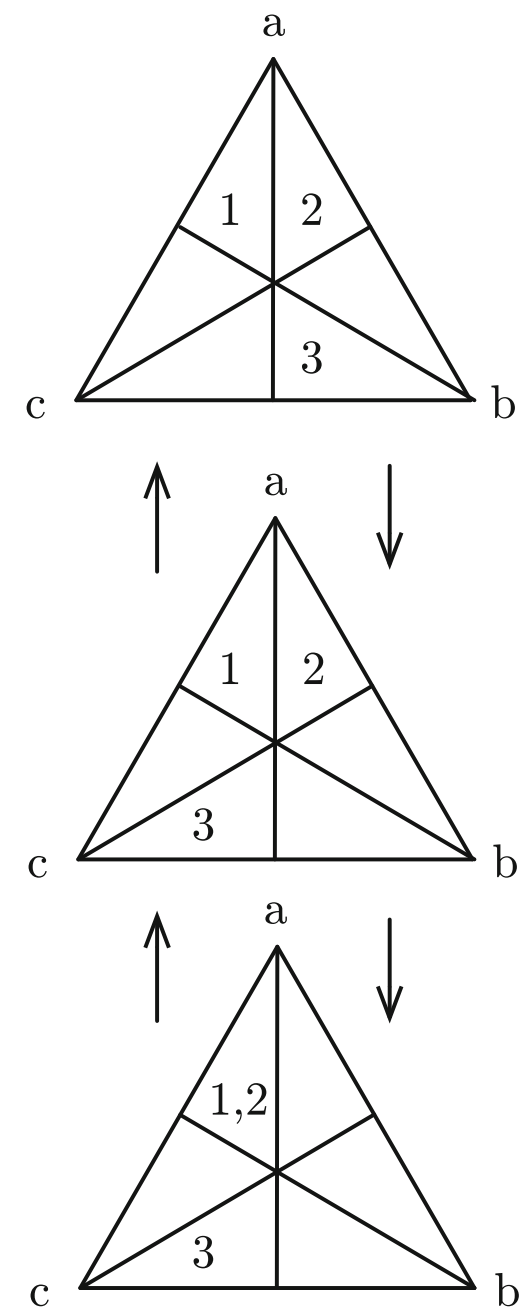

From these inequalities, we can conclude that $h^{1}(a, b)=h^{2}(a, b)=h^{3}(a, b)=$ $h^{\text {central }}(a, b)$.

Combining this with our earlier analysis, we can conclude that $h$ assigns to same value to $(a, b)$ at every profile in the "completion" represented by Fig. 5.

However, in any logically possible completion, the same conclusion would follow.

Moreover, given that in each possible completion the same "central profile" is present, we know that $h$ assigns the same value to $(a, b)$ across all 27 profiles of interest. This is sufficient to establish that FI is implied.

Theorem 5 If a social welfare function satisfies Proximity* and PC then it satisfies dictatorship*. 


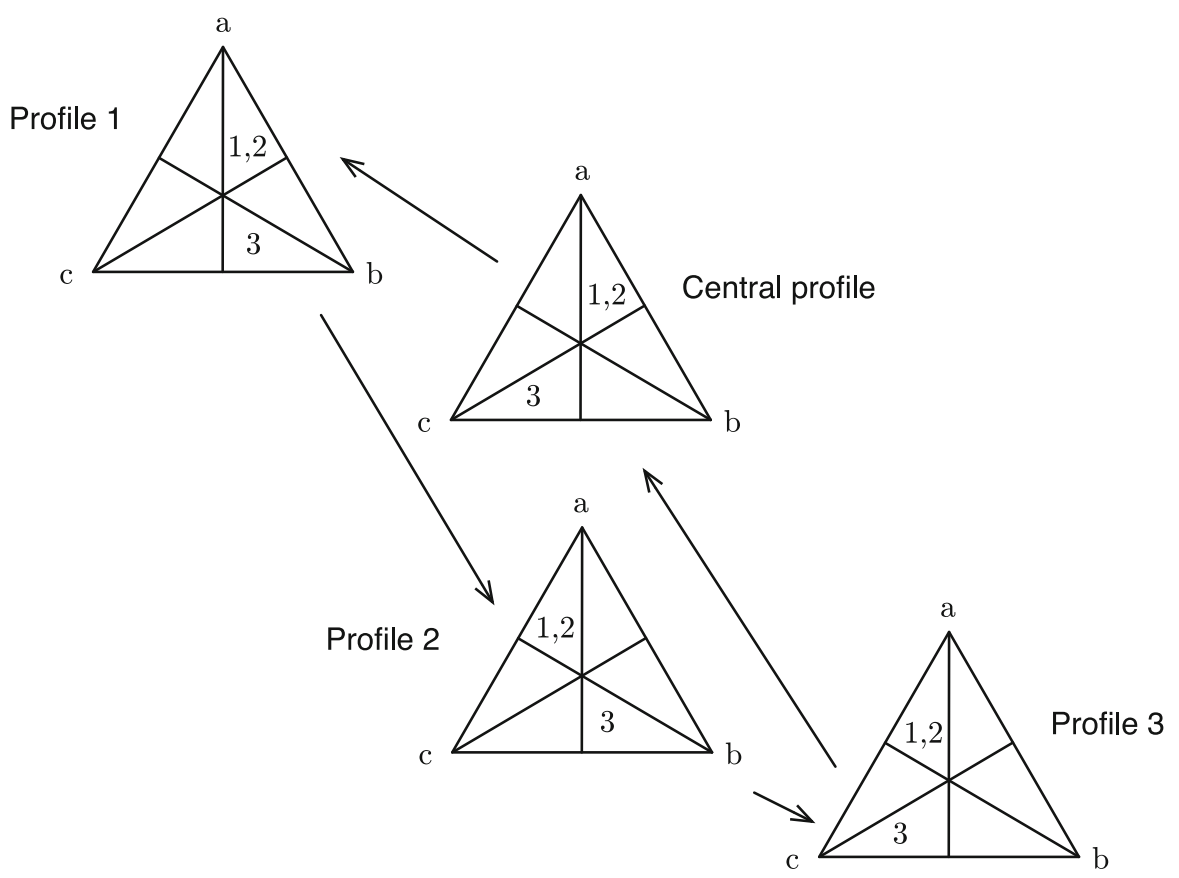

Fig. 8 The middle segment

Proof The domain of a SWF as defined in this paper corresponds to the $H_{E+}^{3}$ domain in Barrett et al. (1986). Their Proposition 2.10 establishes that $H_{E+}^{3}$ is a "non-narrow domain".

Our co-domain $H$ corresponds to the co-domain $H_{+}$in Barrett, Pattanaik and Salles. Their Theorem 3.6 states that every function that maps from a non-narrow domain to $H_{+}$and that satisfies FI and PC is dictatorial. We know, by our Lemma 4, that Proximity* and PC imply FI. Hence, Proximity* and PC imply dictatorship*.

\section{Conclusion}

In this paper we have investigated the social choice implications of the proximity condition. This condition is meant to reflect the normative intuition that when people move closer to you then the social choice should not move further away from you. We have shown that this condition is incompatible with other conditions.

For merging functions that are interval scale invariant, proximity is equivalent to dictatorship. Moreover, for social welfare functions that satisfy the Pareto criterion, Proximity* implies dictatorship*. This means that either proximity is not an attractive normative requirement after all, or we must give up some other social choice condition. Of course, another possibility is that our intuition about proximity needs to be codified using different axioms. We leave this task to future research. 
Acknowledgments We dedicate this paper to Maurice Salles on the occasion of his retirement from the Université de Caen. We are extremely grateful for the comments of Vincent Merlin and an anonymous referee. Financial support from the Spanish Ministry of Science and Innovation through Feder grants SEJ2007-67580-C02-02 and ECO2010-21624, the NUI Galway Millennium Fund and the Irish Research Council for the Humanities and Social Sciences co-funding from the European Commission is gratefully acknowledged.

\section{References}

Aczél J, Roberts FS (1989) On the possible merging functions. Math Soc Sci 17:205-243

Aczél J, Gronau D, Schwaiger J (1994) Increasing solutions of the homogeneity equation and of similar equations. J Math Anal Appl 182:436-464

Arrow KJ (1951) Social choice and individual values. Wiley, New York

Baigent N (1987) Preference proximity and anonymous social choice. Q J Econ 102:161-170

Barrett CR, Pattanaik PK, Salles M (1986) On the structure of fuzzy social welfare functions. Fuzzy Sets Syst 19:1-10

Barrett R, Salles M (2011) Social choice with fuzzy preferences. In: Arrow KJ, Sen A, Suzumura K (eds) Handbook of social choice and welfare, vol 2. North-Holland, Amsterdam

Duddy C, Perote-Peña J, Piggins A (2011) Arrow's theorem and max-star transitivity. Soc Choice Welf $36: 25-34$

Duddy C, Piggins A (2011) A measure of distance between judgment sets. Soc Choice Welf (forthcoming) doi:10.1007/s00355-011-0565-y

Grabisch M, Marichal JL, Mesiar R, Pap E (2009) Aggregation functions. Cambridge University Press, Cambridge

Kemeny JG, Snell JL (1962) Mathematical models in the social sciences. Ginn, New York

MacIntyre IDA (1998) Two-person and majority continuous aggregation in 2-good space in social choice: a note. Theory Decis 44:199-209

Perote-Peña J, Piggins A (2002) Geometry and impossibility. Econ Theory 20:831-836

Quesada A (2007) Merging discrete evaluations. Math Soc Sci 54:25-34

Saari DG (1995) Basic geometry of voting. Springer, Berlin

Saari DG (2001) Chaotic elections!: a mathematician looks at voting. American Mathematical Society, Providence

Salles M (1998) Fuzzy utility. In: Barbera S, Hammond PJ, Seidl C (eds) Handbook of utility theory, vol 1. Kluwer, Dordrecht 